\title{
Functional and parametric mapping of the semantics of phraseological units in intercultural space
}

\section{[Cartographie fonctionnelle et parametrique de la semantique des unites phraseologiques au sein de l'espace interculturel]}

\author{
Didar Ryskulbek - Tynyshtyk Ermekova - Lazzat Alibekova - Saule Bektimirova - \\ Layilya Togzhanova - Peter Ziak
}

DOI: 10.18355/XL.2021.14.04.11

\begin{abstract}
The research objective in the proposed work circles around the semantic of the French phraseological units in the light of the functional-parametric description.

In particular, it identifies the new motivational characteristics of the national language, as well as the linguistic and cultural commentary of phraseological units and the principles of correlating the semantic basics of different systems and sentences based on figurative sense and locutions in the French language.

The study aims to develop a new model of functional cartographic and parametric cartography of the phraseological units in the interlingual and intercultural space and to present a methodology for the development of the phonological unit.
\end{abstract}

Key words: phraseology, units, semantics, intercultural space, signs

\section{Résumé}

L'objet de recherche dans le travail proposé porte sur la sémantique des unités phraséologiques françaises à la lumière de la description fonctionnelle-paramétrique. Le sujet de la recherche est l'identification de nouvelles caractéristiques motivationnelles du sens des expressions figées stables dans la langue nationale, ainsi que le commentaire linguistique et culturel des unités phraséologiques et les moyens de corréler la sémantique des unités de différents systèmes de phrases basés sur les expressions figées de la langue française.

L'objectif de l'étude est de développer un nouveau modèle de cartographie fonctionnelle et paramétrique de la sémantique des unités phraséologiques dans le cadre de l'espace interlingue et interculturel et ainsi proposer une méthodologie pour décrire le sens des unités phraséologiques.

Mots clés : phraséologie, unités lexiales, sémantique, espace interculturel, signes

\section{Introduction}

La pertinence de la recherche est déterminée par la nécessité même de recourir à une analyse approfondie de la sémantique des unités phraséologiques, de rechercher diverses approches pour l'étude des spécificités des unités phraséologiques en tant que signes de langue et de culture. La recherche entreprise montre comment diverses composantes de la structure sémantique des unités phraséologiques (dénotatives, évaluatives, motivationnelles, émotives) interagissent les unes avec les autres, car une signification culturelle particulière dans la sémantique des unités phraséologiques est en corrélation avec d'autres niveaux de sens (et, surtout, avec la forme interne des unités phraséologiques). Les images des unités phraséologiques 
présentent généralement en elles l'expression de significations culturelles qui déterminent les spécificités nationales et culturelles des unités phraséologiques. Sur la base du matériel de langue, l'opportunité de présenter un nouveau modèle de description de la sémantique des unités phraséologiques est présenté, afin de généraliser et de compléter les connaissances existantes dans le domaine de la linguistique théorique et appliquée sur les origines et l'interprétation des unités phraséologiques.

Dans les symboles, le lien entre le signifié et le signifiant est déterminé arbitrairement. Par convention, ils sont donc appelés signes conventionnels.

Les signes iconiques et index sont dits immédiats ou naturels, car en eux la relation entre le signifiant et le signifié semble être motivée par l'essence des deux côtés du signe.

Si nous observons les signes d'une langue naturelle du point de vue de la signification des unités phraséologiques, vous pouvez voir que non seulement les symboles, mais aussi les indices et les signes iconiques fonctionnent dans la langue au niveau phraséologique. Par exemple, c'est dans le phénomène du corps humain que certains concepts trouvent la forme de leur matérialisation. Ces changements on peut voir dans les unités phraséologiques qui se reflète la conceptualisation des gestes humains et des mouvements de son corps.

« La prise en compte d'unités phraséologiques avec des composants désignant des parties non détachables du corps nous permet d'affirmer qu'elles sont souvent basées sur des gestes, des expressions faciales, des mouvements corporels associés aux réactions d'une personne au comportement des autres et au monde qui l'entoure. Ces réactions physiques sont sans aucun doute similaires chez les personnes appartenant à des communautés linguistiques différentes, contrairement aux coutumes, aux rituels, à la symbolisation de phénomènes naturels et de réalités diverses, qui sont des facteurs de culture reflétés dans la langue. La question de savoir comment les réactions du corps humain peuvent être exprimées verbalement dépend des locuteurs de la langue et, bien sûr, des capacités de la langue elle-même, et cela peut souvent être accidentel. Pour cette raison, lors de la transmission de réactions physiques en réponse au comportement d'autres personnes, à divers types de phénomènes naturels et à des situations inadéquates, la phraséologisation est très souvent utilisée. En effet, tant que la description d'une situation n'est pas devenue un signe, elle ne peut être comprise sans ambiguïté par tous les membres de la communauté linguistique, sans parler des locuteurs d'une autre langue. Ce n'est qu'alors que la sensation véhiculée par les mots peut devenir compréhensible pour tous les membres de la communauté linguistique, sans parler des locuteurs d'une autre langue » (Saussure, 1989).

Le PU de type iconique peut être attribué à la conceptualisation suivante des mouvements gestuels d'une personne et des actions de son corps :

1) secouez la tête - « surprise », « désaccord »;

2) taper du pied - « rage »;

3) rire aux larmes - « s'amuser »;

4) montrer le blanc des yeux - faire des yeux effrayants - «faire peur»; 
5) claquer la porte - claquer la porte - « insatisfaction, rupture des relations» ;

6) rire à gorge déployée - rire à tue-tête - « fort »;

Les unités phraséologiques ci-dessus sont généralement facilement comprises par les locuteurs natifs et les étrangers qui apprennent cette langue. Cela est dû au fait que, ressentant des réactions physiologiques similaires sur eux-mêmes dans certaines circonstances de la vie, ils peuvent déterminer approximativement quelles émotions et sentiments expriment certaines sensations, gestes et réactions.

«Avec l'approche cognitive, la propriété d'iconicité n'est plus associée à des signes individuels, mais à la structure de la langue dans son ensemble et ses divers aspects», explique Kobozeva (Kobozeva 2000). «L'iconicité est comprise comme la correspondance de la structure du langage avec cette structure conceptuelle du monde réel, qui s'est formée dans l'esprit humain sur la base des données de l'expérience » (Humboldt, 1987).

Se référant aux données pratiques des études typologiques des classifications linguistiques naturelles des plantes et des animaux, B. Berlin distingue clairement trois niveaux, qu'il appelle « le niveau des formes vivantes, le niveau générique et le niveau espèce» (Berlin, 2003).

Il convient de noter que les particules du niveau générique sont désignées par des mots simples et les éléments du niveau spécifique sont désignés par des phrases, mais aussi sur les développements du psychologue E. Roche, qui a prouvé que dans la mémoire humaine il existe des concepts qui représentent le niveau de base de catégorisation des objets du monde objectif. Selon B. Berlin - le niveau générique, et que les concepts du niveau des espèces est plus complexe.

I. Kobozeva estime que « la motivation iconique est la correspondance des relations entre des parties d'une structure linguistique et des parties d'une structure conceptuelle qui reflète la réalité. Les manifestations de la motivation iconique sont multiples. Ainsi, l'opposition des signes simples aux signes complexes du même niveau de langue peut être considérée comme le reflet de la simplicité/complexité des concepts qui leur correspondent dans une langue donnée. Si, par exemple, en russe, le concept de "vapeur" est exprimé par le simple mot vapeur, et le concept de "locomotive à vapeur" est exprimé par le mot complexe locomotive à vapeur, alors selon l'hypothèse sur l'iconicité de la langue, cela signifie que pour les locuteurs natifs de la langue russe, le premier concept est en un sens plus élémentaire que le second, initial par rapport à lui » (Kobozeva, 2001). Ainsi, la structure sémantique du signifiant se trouve motivée par la structure du signifié. Un concept simple correspond à une expression, à un mot, et un concept dérivé correspond à une expression, à un mot ; tandis que plus les signifiés sont étroitement liés, plus le lien entre les signifiants dans la composition d'un signe linguistique complexe est fort.

$\mathrm{Au}$ cercle des phénomènes linguistiques où se trouve l'iconicité, on peut aussi attribuer des unités phraséologiques, où la structure du signifié est associée à la structure du signification. 
Précédemment, MM Kopylenko a conceptualisé la spécificité nominative des unités phraséologiques qui a conduit à la formulation d'un problème théorique important de distinction entre conceptualité absolue et non absolue. L'auteur considère les concepts formés comme absolus, ce qui correspond à un certain phénomène linguistique - un mot simple, par exemple « table » ou « garçon ». Toutes les autres formations linguistiques correspondent à une conceptualité non absolue, qui diffère en degrés. La mesure de la conceptualité dans la sémantique des combinaisons de phrases est moindre que dans la sémantique des mots. Par conséquent, ils reflètent le moindre degré de conceptualité. Selon MM Kopylenko Popova «Le degré de conceptualité peut être déterminé selon l'intégrité de la forme linguistique, selon son démembrement et l'utilité sémantique ou l'infériorité des composants ». En d'autres termes, moins l'indépendance des composants d'une forme linguistique est grande, plus forte est l'intégrité du sens qu'elle exprime, plus elle est conceptuelle. Une telle gradation semble légitime, puisque le concept ne peut être considéré comme quelque chose de donné une fois pour toutes.

L'environnement naturel et l'activité de la parole sont l'essence de la formation de nouvelles fonctions symboliques des noms.

L'activité nominative est un domaine de nouvelles connaissances, "un domaine de supersavoir, c'est-à-dire recombinaison inconsciente de connaissances précédemment accumulées, qui au moment de l'observation révèlent de nouvelles vérités. La formation d'unités nominatives dépend aussi directement de la finalité de son utilisation par le nominateur dans la communication. Autrement dit, la forme, le contenu, la sémantique, le type de nomination sont déterminés par les fonctions cognitives et communicatives des unités correspondantes. Les propriétés d'un signe de langue sont prédéterminées par les fins pour lesquelles ils sont utilisés. En linguistique, le problème des buts, dont la réalisation est assurée par l'usage des signes, est considéré comme le problème des fonctions des langues. La langue remplit un certain ordre de fonctions, dont la principale est la fonction de communication - la fonction d'assurer la communication entre les personnes. Dans la communication linguistique, une certaine entité immatérielle - l'information de connaissance - est transmise d'une personne à une autre indirectement, mais indirectement, à l'aide d'entités imparfaites, qui sont des expressions linguistiques. Ils apparaissent comme le résultat de la prise de conscience de la nécessité de trouver une expression symbolique pour certaines idées, sensations et circonstances situationnelles étroitement liées à une personne, au comportement des personnes dans un certain environnement, aux relations entre les personnes.Une personne apprend le monde et forme sa vision du monde. La cognition s'accompagne d'une évaluation, donc, dans les actes nominatifs, les résultats de l'activité humaine gnoséologique et axiologique sont formalisés au moyen du langage. Dans un acte nominatif, une unité linguistique peut être créée pour remplir une fonction nominative ou évaluative. Les unités nominatives qui véhiculent des éléments de connaissance sur le monde remplissent une fonction nominative, les unités nominatives qui véhiculent une évaluation remplissent une fonction évaluative» (GV Kolshansky, 1990).

L'appel à la capacité de parole-pensée des locuteurs suppose également un recours à la base méthodologique correspondante dans l'étude des fondements conceptuels et 
linguoculturels de la phraséologie : de la pensée à exprimer aux formes linguistiques de son expression, de la pensée conceptuelleactivité réflexive de la conscience à la formation des moyens nominatifs secondaires du langage dans leur finalité communicative.

L'isofonctionnalité du mot et des unités phraséologiques permet de combiner le vocabulaire et la phraséologie dans un système unique et très précieux à bien des égards, en particulier, dans un système de moyens de nomination généralisée, c'està-dire des moyens de représentation, des concepts, des entités cognitives, sans lesquels la pensée, la cognition, la communication, et par là le progrès humain, sont inconcevables.

Du point de vue des sciences cognitives, la nomination est interprétée comme un moyen de représenter des concepts, ou comme la corrélation d'un nom avec un phénomène non linguistique. "Sur les plans fonctionnel et sémiotique, la nomination est une représentation d'idées, de concepts, de jugements, d'inférences à l'aide de moyens linguistiques, la corrélation de signes linguistiques avec les phénomènes correspondants du monde »(Telia, 1999). Si dans les actes de nomination linguistique, les concepts sont transformés en sens lexicaux, alors avec la nomination secondaire comme moyen de matérialisation des concepts, il y a l'accomplissement d'une activité pensante-linguistique productive de la conscience, procédant sous l'influence du contenu conceptuel déjà assigné au mot donné.

\section{Résultats}

Toutes les tâches présentées dans notre travail sont principalement liées à la cartographie fonctionnelle-paramétrique de la sémantique des unités phraséologiques et se réduisent aux suivantes :

- justifier l'opportunité d'une description fonctionnelle-paramétrique des unités phraséologiques ;

- décrire le fonctionnement des macrocomposantes des unités phraséologiques telles que dénotatives, grammaticales, évaluatives, motivationnelles, émotives, stylistiques séparément et dans leur interaction ;

- déterminer le contenu de la composante motivationnelle dans la sémantique des unités phraséologiques : mettre en évidence le fondement, le contenu et les critères de systématisation pour motiver le sens du roulement durable ;

- étayer et présenter la structure d'une nouvelle composante de la sémantique des unités phraséologiques - linguoculturelle, sur cette base, proposer un commentaire linguoculturel des unités phraséologiques ;

La nouveauté scientifique de la recherche réside dans le fait que le rôle principal dans la structure sémantique des unités phraséologiques est attribué à la composante motivationnelle, qui détermine d'autres composantes de la sémantique des unités phraséologiques. À cet égard, pour la première fois, la nature intralinguistique et extralinguistique de la phraséogenèse a été formulée et décrite comme un critère formant système au cours de la motivation pour le sens des expressions figées. A la lumière du paramètre motivationnel, les versions de l'origine des unités 
phraséologiques dans le cadre des hypothèses et des présomptions sont caractérisées pour la première fois.

Essayons de déterminer les propriétés d'un signe phraséologique.

\section{Méthodes de recherche}

Les tâches décrites ont nécessité l'utilisation de nombreuses méthodes de recherche linguistique, en particulier, composante, appositive, distributive, étymologique, etc. Chacune de ces méthodes est utilisée dans son domaine : la méthode d'analyse en composantes est utilisée pour déterminer la valeur significative d'une unité phraséologique ; la méthode de description fonctionnelle-paramétrique - lors de la détermination de la structure sémantique des unités phraséologiques; méthode cognitive - lors de la clarification de la forme interne d'une unité phraséologique, son complexe figuratif associatif de caractéristiques associées à la capacité d'un individu à représenter des situations du monde réel (moins souvent - fictif), reflétées dans la conscience; méthode étymologique - lors de la restauration de l'image originale de la forme interne et de la motivation historique des significations des unités phraséologiques; la méthode contrastive - lors de la comparaison d'unités phraséologiques de différentes langues qui sont corrélées dans le sens. Cette dernière méthode permet de retracer à la fois les schémas généraux et les similitudes de sens des unités phraséologiques de différentes langues, et leurs différences : dénotatives, grammaticales, axiologiques (évaluatives), motivationnelles, émotives, stylistiques.

D'autres méthodes d'analyse linguistique ont été utilisées en fonction d'une tâche particulière. Par exemple, pour montrer le fonctionnement réel des unités phraséologiques, des méthodes distributives et contextuelles ont été utilisées. Pour l'analyse linguoculturologique des unités phraséologiques des langues russe et française, un commentaire a été principalement utilisé, dont la structure a été développée par un groupe d'auteurs (voir [Grand dictionnaire phraséologique de la langue russe. Signification. Utilisation. Commentaire culturel sous la direction de VN Telia] Il y a eu une comparaison sémantique-diachronique des « images du monde», programmées à l'origine dans le langage (Vorobiev, 2003).

Les méthodes ci-dessus de recherche linguistique de la sémantique des unités phraséologiques n'ont pas été utilisées à «parts égales », leur application a été dictée par un certain degré de nécessité et de commodité. La source du matériel de recherche était le dossier de l'auteur, qui est une sélection ciblée de divers dictionnaires (unilingues et bilingues) phraséologiques, linguistiques, culturels, linguistiques, étymologiques et autres. Sur la base d'une large compréhension des unités phraséologiques. Au total, environ 8000 phrases stables ont été analysées, mais une attention particulière a été accordée aux unités phraséologiques idiomatiques, car c'est en elles que la composante nationale et culturelle est la plus clairement représentée.

La base théorique de la recherche était à la fois des travaux liés à la période "classique" (Vinogradov, 1999) de la formation de la phraséologie en tant que discipline linguistique spéciale, et les travaux de la dernière décennie du XXème et de la première décennie du XXIème siècle: le développement de nouveaux problèmes, en particulier, la méthode de description cognitive, fonctionnelle et 
paramétrique des unités phraséologiques, ainsi que la formulation des principales dispositions de fond de la direction linguoculturologique en phraséologie.

\section{Discussion}

I.Ray un phraséologue français identifie les propriétés suivantes d'un signe phraséologique :

1) La polylexicalité;

2) La fréquence;

3) Le figement ou fixité;

4) Le défigement, désautomatisation ou délexicalisation;

5) L'institutionnalisation (introduction aux dictionnaires) ;

6) L'idiomacité;

7) La figuralité;

8) L'iconicité;

9) L'opacité;

10) L'ambiguïté;

11) L'écart ou déviation;

12) La moulabilité ou productibilité;

13) La répétition;

14) La reproduction;

15) Les différents régistres;

16) La réductibilité;

17) L'arbitrariété, la motivation et la démotivation;

18) La valeur métaphorique;

19) La remétaphorisation;

20) Les éléments expressifs et les procédés productifs (V Humboldt, 2010).

I.Ray comprend la polylexicalité comme des constructions syntaxiques distinctes. Les mots sont graphiquement séparés les uns des autres, malgré le fait que $\mathrm{F}$. Saussure ait attiré l'attention sur quelques cas d'agglutination dans la langue française, c'est-à-dire fusionnant en un seul symbole graphique de plusieurs composants auparavant autonomes. On remarque que la langue française vise non seulement la condensation sémantique, mais aussi formelle (la fusion des mots) : tous jours $\rightarrow$ toujours, au jour d'hui $\rightarrow$ aujourd'hui. Les limites de la polylexicalité sont indiquées par les phraséologues français à partir du minimum : voir rouge;

avant: aller où le roi ne va pas qu'à pied, en avril ne te découvre pas d'un fil;

avant les textes (maximes): il n'y a pas un point d'accidents si malheureux dont les gens habiles ne tirent que quelques avantages (La Rochefoucauld);

prêter de l'argent fait perdre la mémoire;

les proverbes: à bon chat, bon rat;

aphorismes, axiomes, théorèmes : le tout est plus grand que sa partie, etc.). 
La propriété de particularité est nécessaire, selon I. Ray, pour déterminer la frontière entre les combinaisons libres et non libres. Dans quels types de textes et de contextes et à quelle fréquence tel ou tel sens apparaît-il. C'est-à-dire que la combinaison de mots dans des combinaisons et des séquences répétitives détermine non seulement la stabilité sémantique et formelle, mais aussi la stabilité discursive.

Si vous essayez de résumer les propriétés d'un signe phraséologique, il se distingue par:

a) Conception séparée ;

b) Une combinaison de mots avec des significations différentes ;

c) Conformité avec le prototype phraséologique ;

d) Cohérence de la forme interne ;

e) diffusion ;

f) Étendue et sémantique non détaillée ;

g) Une large base sémantique, qui permet d'appliquer une unité phraséologique à une large classe de sujets ;

h) Caractéristique évaluative (les signes du phrasème sont le résultat et le moyen de la pensée émotionnelle, activité de qualification-évaluation de la conscience);

i) Ne pas nommer le sujet, mais comprendre la situation.

Le développement de ce point de vue des sciences cognitives semble prometteur à la fois pour la théorie de la nomination phraséologique, la sémantique phraséologique, et pour approfondir l'étude des relations entre langage et pensée en général.

Il semble que du point de vue actuel, le problème de la signification d'un signe phraséologique devrait être formulé : quelle est la base conceptuelle ou cognitive du signe, combien d'informations sont allouées par le corpus du signe à partir du bagage de connaissances à propos de l'environnement ? Il est nécessaire d'identifier les structures qui sous-tendent la compréhension de la sémantique phraséologique : une telle identification est pertinente pour les tâches des sciences cognitives. Il éclaire également le concept de perception du monde.

Nous partirons du fait que la perception d'un objet signifie non seulement une séparation perceptive, mais aussi conceptuelle de l'environnement des autres objets, c'est-à-dire donner à l'objet une certaine signification ou un certain concept comme sa représentation mentale, une image conceptuelle, indiquant que le sujet a un certain état intentionnel de perception. Cette construction de concepts de signesobjets se caractérise par le fait que de nouvelles significations se construisent à partir de celles existantes. De plus, l'activité mentale d'une personne choisit les entités susceptibles de s'appliquer à toute une gamme de phénomènes homogènes, quelle que soit la période de leur existence réelle. La désignation des choses sous une forme généralisée, compte tenu de leurs caractéristiques universelles et essentielles, s'appelle une nomination dans les unités linguistiques correspondantes.L'attention portée aux phénomènes de signification des unités phraséologiques est symptomatique du stade moderne de la recherche linguistique. Elle s'explique en partie par l'inefficacité de considérer les problématiques sémantiques de la phraséologie sans tenir compte de l'analyse des processus de perception et de cognition des objets et des états du monde, de la compréhension et de l'usage du langage. 
Lors de la traduction de réactions en réponse au comportement d'autrui, de réactions à des phénomènes incontrôlables et à des situations inadéquates de la nature, à l'aide du langage, les situations verbalisées sont très souvent phraséologisées. Puisque jusqu'à ce que la reconstruction d'une situation particulière devienne un signe, elle ne peut pas être comprise de manière adéquate par le reste de la communauté linguoethnique, ainsi que par les locuteurs d'une autre langue. Un sentiment dépeint verbalement peut devenir sans ambiguïté et compréhensible pour tous les membres de la communauté ethnolinguistique lorsqu'un tel signe, comme unité phraséologique, lui est trouvé. Les unités phraséologiques peuvent véhiculer une situation à l'aide de mots - composants qui ont perdu les liens avec leur signifié dénotatif non seulement à l'aide d'une image, mais aussi à l'aide d'un syntagme devenu signe linguistique.

Ainsi, le langage remplit sa fonction principale en raison de la connexion conditionnelle et stable entre le signifiant et le signifié. L'aspect nominatif de la fonction signe du langage est la composante où la réalité réfléchie se présente sous la forme du signifié et où l'instantané épistémologique d'un fragment de réalité reçoit un démembrement conceptual-linguistique, significatif. L'économie d'une langue, qui est une de ses propriétés essentielles, oblige les langues à éviter un accroissement quantitatif d'unités de son plan d'expression et fait de l'activité nominative une nomination secondaire - à repenser les moyens nominatifs déjà disponibles dans la langue.

En résumant ce qui précède, on peut dire que l'acte de nomination agit comme une action discours-pensée, dont on ne peut ôter un lien intermédiaire : cognitif, réflexif, conceptual-logique ou figuratif, confirmant l'inclusion de l'événement ou de la situation à nommer dans la vie d'une personne.

Procéduralement, le rapport entre pensée, réalité et langage apparaît dans une nomination indirecte dans une interaction complexe et multiforme. Le sens même est un phénomène spirituel, c'est la dépendance informationnelle des concepts de deux choses dans l'esprit. Le sens surgit chaque fois que l'un informe l'autre et ajuste la conscience à cette seconde en tant qu'information importante. Le sens existe dans des relations conceptuelles d'un certain type. Les concepts en tant qu'essences discrètes et significatives de la conscience sont unis par des liens conceptuels.

Ces connexions sont de trois sortes : implication, comparaison-classification et sémiotique (signes). Les premiers sont une analogie mentale des connexions réelles des entités du monde objectif. En fin de compte, c'est un reflet des connexions entre les choses, les événements, entre la partie et le tout, entre la chose et les signes. Un concept présuppose, évoque la pensée d'un autre, un concept en implique un autre du fait de la dépendance, de l'interaction, de la connexion des entités qu'ils reflètent. L'exemple le plus évident de relations d'implication est le reflet des relations de cause à effet, mais cela inclut également les relations spatiales, temporelles, holopartitives et autres, c'est-à-dire des connecteurs linéaires d'un certain genre, contiguïté, consistance, conjugaison.

L'implication peut être le reflet des cas les plus divers de concurrence d'entités : simultanée et multitemporelle, statique et dynamique, mutuellement directionnelle et 
unidirectionnelle, etc. Un point important est à noter ici : les liens des entités réfléchies peuvent être unidirectionnels et mutuels, mais les liens conceptuels sont toujours mutuels, réversibles. Par exemple, non seulement la pensée d'une cause présuppose la pensée d'un effet, mais aussi l'inverse. Il suffit d'activer le réglage informationnel de la conscience, et les connexions d'implication étonnent le phénomène du sens. Un concept en actualise un autre, important sur le plan informationnel, et ce second s'avère être le sens du premier. Un autre type important de connexions conceptuelles qui structurent la conscience sont les connexions de classification comparative. Ils sont une analogie mentale de la distribution des attributs dans les entités du monde. En d'autres termes, la base objective de comparaison - la classification est la communauté - les différences entre les essences du monde reflétées par la conscience selon les signes qu'elles détectent. Les caractéristiques communes peuvent résider dans le domaine des intentions des concepts comparés, c'est-à-dire qu'elles sont classées comme formatrices de classe, puis les concepts sont construits le long de la verticale spécifique au genre (classification ou hiérarchie hyper-hyponymique des concepts) ou sont construits au même niveau de généralisation que les espèces du même genre (liaison équonymique des concepts). Mais la comparaison peut aller dans le sens des traits secondaires, au moins pour l'un des concepts comparés en termes de contenu, et on a alors affaire à une comparaison au sens étroit du terme (comparaison-similation, assimilation), c'est-à-dire celui qui sert de métaphore principale.

Pour nous, cependant, il est essentiel que les connexions conceptuelles de type classification comparative, fondées sur des traits communs, ne génèrent pas de sens. Et cela se comprend, puisque dans ce cas, la connexion entre les deux entités est établie dans la conscience et ne reflète pas une connexion réelle d'entités, pas leurs dépendances et interactions, mais seulement la communauté de leurs caractéristiques inhérentes ou les niveaux de généralisation des caractéristiques.

Quant aux connexions conceptuelles sémiotiques, les connexions entre les deux faces du signe - le signifiant et le signifié - sont établies à cet effet, afin de porter des significations, et c'est leur différence avec les connexions conceptuelles implicatives. Le signifiant et le signifié ne sont pas liés par le rapport d'implication. Avec une connexion de signe, comme avec une implication, les concepts sont unis par une relation signifiante et le même groupe de sens " moyens ". Le premier d'entre eux actualise le second et ajuste la conscience à ce second comme informationnellement important : le second est le sens du premier. La différence est que pour le signe "signifie" il y a sa fonction, son but, qui ne peut pas être dit à propos des connexions d'implication : un événement signifie quelque chose, parce qu'un sens lui est assigné, mais parce que l'observateur en connaît les implications, et elles méritent son attention.

Ainsi, selon la nature du lien entre les deux concepts, le concept d'actualiser et le concept d'être actualisé, le sens se divise en deux types - implication (non signé) et sémiotique (signe, conventionnel, codifié).

Par rapport au sujet qui nous intéresse, il est important que les signes impliqués euxmêmes portent les deux sortes de sens - le signe (sémiotique) proprement dit et l'implication. Le fait qu'ils portent le premier va de soi : c'est à cela que sert le signe. 
Mais il n'est pas difficile de voir dans le signe actualisé la présence d'un sens du second genre - l'implication. Puisque sa base, la source dans ce cas n'est pas simplement un événement, mais un signe d'événement, alors ce genre de significations d'implication devrait être appelé cette implication par leur source.

En effet, un acte de signe avec toutes ses composantes, à tous les niveaux de sa structure, par lui-même et en interaction avec l'environnement de sa mise en œuvre, sert de source d'implications non-signes, c'est-à-dire qu'il est perçu non seulement comme un signe avec sa signification symbolique, mais est analysé dans son ensemble comme un phénomène intégral, et ainsi une masse d'informations diverses en est extraite. Ces informations complètent d'une certaine manière le sens proprement signifié des déclarations et des textes, d'une certaine manière la complique, et d'une certaine manière la contredit, entre en conflit avec elle et la corrige. En fin de compte, le destinataire extrait un certain résultat résumé de l'interaction du sens sémiotique codifié et non codifié à ces implications des actes de signe. La première découle de la connaissance de la langue, la seconde de la connaissance du monde, des personnes, de l'activité des signes, de la connaissance de l'usage de la langue, et cette différence est significative, malgré le fait qu'il n'y ait pas de frontière rigide entre connaissance de la langue et connaissance du monde d'abord en raison du caractère probabiliste des sens codifiés eux-mêmes.La particularité des implications est qu'elles ne sont pas codifiées. Ils représentent des connaissances inférentielles, des conclusions probabilistes à partir de situations spécifiques d'utilisation des signes. Ils s'appuient sur toute l'expérience humaine, sur des structures complexes de connaissances probabilistes, sur des chaînes ramifiées de causes et d'effets, de coïncidences et de juxtapositions. Ils supposent la connaissance d'une variété de domaines avec leurs connexions et dépendances, dans lesquels ils sont déduits par l'analyse d'implication de l'utilisation de la langue. Ici chaque « signe » a une signification selon les circonstances d'utilisation et n'est donc pas un signe. Il n'y a pas de dictionnaire et de grammaire au sens propre, mais il existe des connexions et des dépendances probabilistes d'entités et de phénomènes (événements) dans les mondes réel et imaginaire reflétés par la conscience et créés par elle. Même avec une dépendance rigide (sans ambiguïté) et hautement probable d'un événement à un autre, le premier ne peut être considéré comme un signe du second, à moins qu'il n'y ait un expéditeur derrière lui avec l'intention de communiquer un sens.

Pour notre propos, cependant, il est essentiel que le sens d'implication d'un événement se déplace librement dans le champ de ses connexions linéaires avec d'autres événements, et chaque fois que son contenu est déterminé par les circonstances de l'événement, mais la liaison par ce conditionnel plutôt rigide, liaison de l'un à l'autre qui existe entre le signifié et le signe signifiant. La liaison du signifié au signifiant doit donc être rigide, car elle est, en principe, arbitraire et conditionnelle.

L'interprétation sémiotique des choses et des événements comme signifiants est dénuée de sens et redondante parce qu'elle assimile la langue au monde, la connaissance de la langue à la connaissance du monde. Ensuite, il s'avère qu'il existe 
autant de langues qu'il y a de circonstances différentes d'utilisation du signe. Dans le cadre du système de signes accepté, le signe doit adhérer à un certain sens, en le variant, selon les règles de dérivation sémantique. Le signe ne peut modifier radicalement son contenu en fonction des circonstances de son utilisation. Autant qu'il peut leur résister, et cédant à leur diktat, il perd la qualité du signe, et alors il n'y a pas besoin, improductivement, de l'analyser en fonction du signe. Seule la base des implications reste du signe, il perd son essence de signe et devient juste un événement, multipliant leur nombre et s'intégrant dans la structure de leurs connexions. Dans cette structure, il est significatif, mais ne signe plus. Il va au-delà de la langue où son sens a été codifié et maintenant pour établir son sens. Vous devez connaître non pas le vocabulaire et la grammaire de la langue, qui sont devenus parallèles au monde, mais le « vocabulaire et la grammaire » du monde, qui incluent des événements importants dans le cadre et la continuation de la structure de ses connexions.

L'essence du problème est que non pas les événements doivent être réduits à des signes, mais au contraire, les signes doivent être étendus à des événements. Les événements peuvent acquérir un caractère de signe (être sémiotique), mais seulement, comme nous le verrons plus loin, dans des conditions particulières : dans les signes, en plus de leur essence spécifiquement signe, une composante événementielle (matérielle) générique est constamment présente. Pour cette raison, les significations d'implication des signes ne peuvent être précisées dans un dictionnaire, ni par une liste rigide, ni en indiquant les modèles de variation. Ils sont variables dans la mesure où les connexions et les dépendances des entités et des événements du monde sont diverses. La sémiotique est impuissante à dire quoi que ce soit de significatif à leur sujet, si ce n'est qu'il s'agit d'implications de signes en tant qu'événements. Ici, il doit céder la place à ces domaines de connaissances, ordinaires ou académiques (scientifiques), qui sont à l'origine de certains liens spécifiques.

La sémiotisation possible des choses et des événements est une autre affaire. Une chose, une substance ou un événement est sémiotique si elle est spécialement créée pour qu'un signe (par exemple, divers pointeurs, signaux, comme des sémaphores, etc.), ayant d'autres fonctions, soit adapté et utilisé dans une fonction signe. Dans ce cas, ils se débrouillent souvent avec l'image de la chose correspondante (comparez, par exemple, des panneaux peints sur des bancs, des ateliers, etc). La sémiotisation d'une chose, pour laquelle la fonction de signe est complémentaire, est secondaire par rapport à sa finalité principale et directe, en fait un symbole.Ci-dessus a été évoqué le fait bien connu de l'iconicité possible du symbole-similarité entre le symbole et sa dénotation (comparez la roue de la Fortune comme symbole des vicissitudes du destin, la balance aux mains de Thémis comme symbole d'un évaluation équilibrée de la culpabilité, de l'image royale de l'aigle et de sa suprématie - au sens propre comme au figuré - en tant que symbole du pouvoir souverain). Le symbole est toujours motivé, du moins à la fin (avec le temps, la motivation peut s'obscurcir et se perdre), et il est motivé, en premier lieu, par les dépendances d'implication de la chose en cours de sémiotisation. Mais rien ne l'empêche d'être ironique en plus, si le sens recherché permet de conjuguer implication et similitude. Ce qui est important, c'est que l'implication d'une chose à 
ses signes et connexions est obligatoire pour la sémantisation de tout symbole, y compris l'iconique. En fait, la roue tourne, et cela signifie un changement de position (implication), en ce sens c'est comme le destin (simulation). L'aigle a une apparence royale et habite dans les zones les plus montagneuses (implications), et en cela il est comme le souverain suprême (simulation). La balance vibre sous la charge et indique son poids exact (implications), le tribunal doit donc peser toutes les circonstances de l'affaire et la trancher équitablement.

Ainsi, la sémantisation des symboles iconiques repose sur des associations de même type d'implication, et la similitude en plus, par modélisation, oriente le processus de recherche de sens : une chose (un événement) ne véhicule pas seulement ses signes et ses connexions à la dénotation d'un symbole, mais lui sert aussi de modèle. Ainsi, ce qui a été dit à propos des symboles d'implication est également vrai en ce qui concerne les symboles similatifs (iconiques), lorsqu'une chose (un événement) ou son image signifie quelque chose de similaire à elle-même, mais, en règle générale, d'un abstrait plus complexe et moins visuel.

Ainsi, pour que la dépendance implicite des choses et des événements d'une situation simplement significative pour l'observateur se transforme en une situation signifiante, il faut qu'elle « tombe entre les mains » de l'expéditeur de l'information, afin qu'elle puisse être contrôlé, invoqué et créé par lui comme une action intentionnelle d'information et de communication... Une chose-implicatrice de son environnement naturel, pour l'opposer et la mettre en parallèle avec l'environnement comme sa réflexion et son expression information-communicative. Un indicateur de retrait est la capacité de remplacer la chose-implicatrice par un substitut pictural ou autre conventionnel, économiquement et communicativement plus pratique. La substitution est possible, puisque la chose-symbole nous intéresse non pas en qualité naturelle, mais en qualité informationnelle, comme source d'associations nécessaires, simulateur et actualisateur de sens, et nous pouvons la gérer à ce titre.

Les symboles apparaissent dans les limites dans lesquelles une personne peut contrôler la création de choses et d'événements en tant qu'implicateurs de significations. Mais un symbole est un signe, et en tant que signe il requiert un implicateur de son environnement naturel, des « cadres » du monde matériel, une transition et un monde parallèle de désignations de ce monde. Cependant, l'expéditeur a une autre occasion d'actualiser les significations dans la tête de l'observateur au moyen d'implications, sans recourir à des signes. Vous pouvez jouer, créer la situation d'implication souhaitée, donnant l'impression qu'elle surgit d'elle-même, quelle que soit la volonté de l'expéditeur. Après y avoir réagi, l'observateur en extraira la valeur dont l'expéditeur a besoin. Pour le dire simplement, il a la pensée requise, la représentation requise.

La codification sémiotique d'un signe ne peut changer radicalement lorsqu'il désigne et décrit des situations différentes, elle ne varie que dans certaines limites et selon certains modèles. Décrivant le monde, le langage suit le changement des situations, changeant les signes, mais pas les significations des signes (cela n'est possible que dans certaines limites et selon certains modèles de dérivation sémantique). Le signe 
est précieux pour la stabilité de sa signification. Au contraire, les valeurs d'implication, y compris celles à cette implication, ne sont pas stables. Elles dépendent de la situation et sont déduites des circonstances de l'événement ou de l'usage du signe. Le signe est précédé d'une convention sur le sens qui surgit spontanément dans le cas de la genèse d'une langue naturelle dans le cas des signes secondaires - les codes. Par conséquent, le signe requiert le sens de la langue. La valeur est plus large qu'un signe : il n'y a pas de signe sans valeur, mais l'inverse n'est pas vrai. Les significations dérivent non seulement des signes grâce à la connaissance de la langue, mais aussi des événements grâce à la connaissance du monde.Un signe authentique n'implique pas seulement le destinataire, mais encore plus l'expéditeur, malgré le fait que tous deux ont une langue ou un code commun. Le signe est intentionnel, c'est-à-dire met en œuvre l'intention d'information et de communication de l'expéditeur qui l'exécute. Un seul interprète (observateur) ne suffit toujours pas pour considérer un événement significatif pour lui comme un signe. Les choses et les événements se déplacent vers le signe lorsque derrière eux se trouve leur exécution intentionnelle afin d'actualiser les implications qui leur sont associées dans l'esprit de l'observateur. Sur ce chemin, il y a sans aucun doute des gradations intermédiaires dans l'amélioration du symbolisme.

En général, cette échelle s'ouvre sur une chaîne de deux événements dépendants, où il n'y a ni exécuteur ni interprète. Il n'y a pas de signes ou de significations ici. Puis l'interprète apparaît, et avec lui le sens : le premier événement l'informe du second, et dans d'autres cas il n'a pas besoin d'observer le début d'un événement significatif, il suffit d'observer l'événement significatif et de connaître le lien entre les deux événements. C'est le niveau pré-signe d'obtention des valeurs. Ensuite, l'interprète apparaît sur la scène, organisateur d'un événement significatif, visant à générer des situations significatives. A ce niveau, il a une intention d'information et de communication, le but de communiquer du sens, mais il n'y a pas d'outil particulier, un langage pour transmettre des sens, et il utilise le "langage du monde", créant, agissant, poussant sur du sens situations par la connaissance des dépendances d'implication des choses et des événements. C'est le niveau pré-signé de transfert de valeurs.

À l'aide du tableau du célèbre psychologue cognitif Jean Kosterman (Kosterman, 1987) pour la nomination primaire, nous tenterons de le compléter et de retracer à quoi ressemble l'activité spontanée de la dérivation sémantique de la nomination secondaire dans notre « vie cognitive ».

Comment s'effectue la transformation indirecte de la pensée en signes d'une nomination secondaire ? À l'aide du tableau, nous essaierons de tracer le chemin de la conversion d'une idée en un nom secondaire.

\section{Conclusion}

La signification théorique réside dans la justification d'une approche systématique de l'étude de la sémantique des unités phraséologiques; en combinant l'analyse sémantique et linguoculturelle des unités phraséologiques pour décrire la sémantique linguistique et culturelle des unités phraséologiques ; dans la généralisation des 
mécanismes de la phraséogenèse ; dans le développement des fondements théoriques et pratiques du système des équivalents phraséologiques interlingues.

L'importance pratique du travail est visible dans le fait que le matériel et les résultats du travail peuvent être utilisés dans les disciplines académiques des programmes d'enseignement de base dans la préparation des baccalauréats et des maîtrises dans le sens de la "linguistique", dans le travail universitaire cours spéciaux sur la typologie comparée des langues russe et française. Le développement de paramètres sémantiques supplémentaires d'unités phraséologiques peut être important pour la théorie et la pratique de la phraséographie, la création de dictionnaires explicatifs, bilingues, linguistiques-culturels-logiques et autres.

\section{Bibliographic references}

ABDELLI, D.2017. Le savoir phrastique comme entrée à l'écrit en didactique intégrée. In: Synergies Europe, Vol. 12, pp. 39-49. ISSN 1951-6088 ISSN en ligne 2260-653X

ALSHTAIWI, M.2020. Extraction des termes sur la COVID-19 et leurs emplois sémanticosyntaxiques à partir d'un corpus spécialisé. In: Synergies Algérie. ISSN 1961-9472

BENABID, F. 2021. Lexicovid-19, une floraison de nouveautés linguistiques. In: Synergies Algérie, Vol. 29, 2021, pp. 161-175. ISSN 1958-5160 ISSN

BEREZIN, M.F. 2014 Linguistique générale: Manuel pour les universités. In : M.F. Berezin et V.I. Golovine. Moscow : Alliance, $416 \mathrm{p}$.

BOLDYREV, N.N. 2015. Essence anthropocentrique du langage dans ses fonctions, unités et catégories : Questions de linguistique cognitive. n 1, (042). Art. pp. 5-12.

CALVI, S. 2021. L'enseignement/apprentissage des unités phraséologiques en terminologie: un parcours didactique en français L2 sur le commerce international. Synergies Italie, Vol. 17, pp. 109-120. ISSN 1724-0700 ISSN en ligne 2260-8087

DANILENKO, V. 2015. Linguistique générale et histoire de la linguistique : Cours magistral. Moscow : Flinta, $272 \mathrm{p}$.

DANILENKO, V. 2015. Introduction à la linguistique : Un cours magistral. In : V.P. Danilenko. Moscow : Flinta, 288 p.

DESNITSKAYA, A.V. 2004. Linguistique comparée et histoire des langues. In : A.V. Desnitskaïa. Moscow : URSS, 352 p.

GRAFOVA, T.A. - SHAKHANAROVITCH, A.M. - TELIA, V.N. 1991. Académie des sciences de l'URSS, Institut de linguistique. Moscou : Nauka, $214 \mathrm{p}$.

HUMBOLDT, V. 2019. von. Sur la différence entre les organismes du langage humain et l'influence de cette différence sur le développement mental de la race humaine : Introduction à la linguistique générale. Par. avec lui. In : V. von Humboldt. Moscow : KD Librokom, 376 p.

KAMTCHATNOV, A.M. 2016. Introduction à la linguistique, Manuel. In : A.M. Kamchatnov - N.A. Nikoline. Moscow. IN: Flinta, 232 p.

KASEVITCH, V.B. 2013. Introduction à la linguistique : Manuel. Moscow In: Académie, KATSNELSON, S.D. 2010. Linguistique générale et typologique. Moscow : KD Librokom, $344 \mathrm{p}$.

KAREK,M. 2019. L'acquisition de la compétence lexicale à l'école moyenne en Algérie : problèmes liés aux consignes. In: Synergies Algérie, Vol. 27, pp. 15-31. ISSN 1958-5160

KHALIFA, R.A. 2015. La phraséologie du discours juridique français. Étude de cas: les arrêts de la Cour de cassation. In: Synergies Espagne, Vol. 8, pp. 49-64.

KOLSHANSKY, G.V.1990. Image objective du monde dans la connaissance et la langue. Moscow: Nauka, 108 p.

DIVERSITE DES LANGUES ET REPRESENTATIONS COGNITIVES. 1997. Paris : Ophrys, 283 p. 
KOBOZEVA, I.M. 2000. Sémantique linguistique: Un manuel. Moscow : Éditorial URSS, $352 \mathrm{p}$.

KODOUKHOV, V.I. 2012. Introduction à la linguistique, Manuel pour les universités. Moscow : Alliance, $288 \mathrm{p}$.

KODOUKHOV, V.I. 2013. Linguistique générale, Manuel. In : V.I. Kodoukhov; Préface I.A. Tsyganov. Moscow : KD Librokom, 304 p.

KUBRYAKOVA, E.S. 2001. Sur la linguistique cognitive et la sémantique du terme « cognitif », Bulletin de l'Université d'État de Voronej. Série, Linguistique et communication interculturelle. n. 1, C. pp. 4-10.

LAKOFF, G. 1990. Les femmes, le feu et les choses dangereuses, Quelles catégories révèlent sur l'esprit.Chicago et Londres : The University of Chicago Press,

LÉONTIEV, A.N. 1975. Activité. La conscience. Personnalité. Moscow : Politizdat, LUJIĆ, R. 2019. Agir éthique dans les recherches qualitatives en acquisition d'une deuxième langue. In: Synergies Europe. Vol. 14 , pp. 175-190. ISSN 1951-6088

NEROZNAK, V.P. 1996. Personologie linguistique : vers la définition du statut de la discipline : recueil d'articles: travaux scientifiques de Moscou. Etat lingual un-ça. n. 426, pp. 112-116.

PELISSERO, C. 2019. Enaction: principe et moyens dans le cadre du Français Langue

Etrangère. In: Synergies Europe, vol. 14, pp. 159-174. ISSN 1951-6088

POLANI, M. 1985. Connaissances personnelles. Vers une philosophie post-critique. Moscou :

Nauka,

Le facteur humain dans le langage : mécanismes langagiers d'expressivité.

SÉRIDI, H. - BOUDECHICHE, N. 2020. Conscientisation du phénomène collocationnel : de la prise de conscience au savoir-faire langagier. In: Synergies Algérie, Vol. 28, pp.175192. ISSN 1958-5160

TELIA, V.N. - SHAKHOVSKY, V.I. 2008. Théorie linguistique des émotions. Moscow : Gnose

TELIA, V.N. 1999. Tâches prioritaires et problèmes méthodologiques de l'étude de la composition phraséologique de la langue dans le contexte de la culture, Phraséologie dans le contexte de la culture. Moscow : "Langues de la culture russe", pp.13-24.

VENDINA, T.I. 2016. Introduction à la linguistique : Uebnik pour la Licence Académique In : T.I. Vendine. Lyubertsy : Yurayt, 333 p.

VENDINA, T.I. 2010. Introduction à la linguistique: Manuel. Moscow: Lycée, 391 p.

Words: 7607

Characters: 51217 (28,45 standard pages)

Didar Ryskulbek

Prof. Tynyshtyk Ermekova

Kazakh National Women's Teacher Training University

Aiteke bi Street, 99

050000, Almaty

Kazakhstan

Doc. Lazzat Alipbekova

The Eurasian Humanities Institute

M.Zhumabayev avenue, 4

010009 Nur-Sultan

Kazakhstan 
Assoc. prof. Saule Bektemirova Bekmukhamedovna

Al-Farabi Kazakh National University

Al-Farabi Ave., 71

050010, Almaty

Kazakhstan

Lyailya Togzhanova

IT International University

Manas, 34/1

050040 Almaty

Kazakhstan

PhDr. Peter Žiak, PhD.

free-lance researcher

High-school St. Ursula

Nedbalova 4

81101 Bratislava

Slovakia

pepeziak@gmail.com 